\title{
AUTOMORPHISMS FIXING SUBNORMAL SUBGROUPS OF POLYCYCLIC GROUPS
}

\author{
VITTORIO D. ALMAZAR \\ Department of Mathematics, College of Science, University of the Philippines, 1101 Diliman, Quezon City, \\ Phillipines \\ JOHN COSSEY ${ }^{\dagger}$ \\ Mathematics Department, School of Mathematical Sciences, Australian National University, \\ Canberra, 0200, Australia
}

(Received 25 November, 1997)

0. Introduction. We denote by $\operatorname{Aut}_{s n}(G)$ the set of all automorphisms that fix every subnormal subgroup of $G$ setwise. In their paper [5], Franciosi and de Giovanni began the study of $\operatorname{Aut}_{s n}(G)$. Other authors have also considered the structure of $\operatorname{Aut}_{s n}(G)$ under various restrictions on the structure of $G$ (Robinson [11], Cossey [2], Dalle Molle [4]). The inner automorphisms in $\operatorname{Aut}_{s n}(G)$ are precisely the inner automorphisms induced by elements of $\omega(G)$, the Wielandt subgroup of $G$. Recall that the Wielandt subgroup of a group $G$ is the set of all elements of $G$ that normalise each subnormal subgroup of $G$ and that $\zeta(G)$, the centre of $G$, is contained in $\omega(G)$. Thus $\operatorname{Aut}_{s n}(G) \cap \operatorname{Inn}(G)$ is isomorphic to $\omega(G) / \zeta(G)$ and some of the results obtained indicate that the structure of $\operatorname{Aut}_{s n}(G)$ is controlled by the structure of $\omega(G) / \zeta(G)$; for example, Robinson [11, Corollary 3] shows that, for a finite group $G$, $\operatorname{Aut}_{s n}(G)$ is insoluble if and only if $\omega(G)$ is insoluble. We shall prove a result of a similar nature here. One of the main results (Theorem B) of Franciosi and de Giovanni [5] is that, for a polycyclic group $G, \operatorname{Aut}_{s n}(G)$ is either finite or abelian. We shall show that $\operatorname{Aut}_{s n}(G)$ can indeed be infinite, but only if $\omega(G) / \zeta(G)$ is infinite.

TheOREM 1. Let $G$ be a polycyclic group. Then $\operatorname{Aut}_{s n}(G)$ is infinite if and only if $\omega(G) / \zeta(G)$ is infinite.

The Wielandt subgroup of a polycyclic group is close to being central and if $G$ is nilpotent-by-abelian, for example, then $\omega(G) / \zeta(G)$ is finite (Cossey [3, Theorem 1]). We should expect it to be uncommon for a polycyclic group to have $\omega(G) / \zeta(G)$ infinite. However the example in Cossey [3] shows that a polycyclic group $G$ can have $\omega(G) / \zeta(G)$ infinite and hence that $\operatorname{Aut}_{s n}(G)$ can be infinite. Theorem 1 is an immediate corollary of the next result, but we will need to prove Theorem 1 as an intermediate step in the proof.

Theorem 2. Let $G$ be a polycyclic group. Then $\operatorname{Aut}_{s n}(G) \cap \operatorname{Inn}(G)$ has finite index in $\operatorname{Aut}_{s n}(G)$.

†The second author wishes to acknowledge the hospitality and support of the University of the Philippines, Diliman, where the work for this paper was done. 
1. Preliminaries. Before we begin the proof of Theorem 2, we need some technical lemmas. Some of these are presumably known, but we have not yet been able to find them in the form we need and so have included proofs. A polycyclic group $G$ has a unique maximal finite normal subgroup, which we call the torsion radical of $G$ and denote by $\tau(G)$. We shall use frequently and without further comment the fact that a polycyclic group is finite if and only if every element has finite order.

Lemma 1. Let $G$ be a polycyclic group. If $\tau(G)=1$, then $\tau(G / \zeta(G))=1$.

Proof. Put $A / \zeta(G)=\tau(G / \zeta(G))$. Then $\zeta(A)$ has finite index in $A$ and so $A^{\prime}$ is finite. Since $A^{\prime}$ is normal in $G$ we have $A^{\prime}=1$. If $n$ is the order of $A / \zeta(G)$, we have $[a, g]^{n}=\left[a^{n}, g\right]=1$, for all $a \in A, g \in G$. Thus $[A, G]^{n}=1$ and so the normal subgroup $[A, G]$ is finite, giving $[A, G]=1$. We now have $A=\zeta(G)$, as required.

Lemma 2. Let $A$ be a group of automorphisms of the finitely generated group $G$. Suppose that $G$ has a finite A-invariant normal subgroup $N$ such that $A / C_{A}(G / N)$ is finite. Then $A$ is finite.

Proof. Let $g_{1}, \ldots, g_{m}$ be a set of generators for $G$. If $\alpha$ is any element of $C_{A}(G / N)$, then $\alpha\left(g_{i}\right)$ lies in a fixed finite set of elements, for each $i$, namely $g_{i} N$. Since $N$ is finite, it follows that $C_{A}(G / N)$ is also finite. Thus $A$ is finite.

Lemma 3. Let $G$ be a polycyclic group and let $H=G / \tau(G)$. Then $\omega(G) / \zeta(G)$ is finite if and only if $\omega(H) / \zeta(H)$ is finite.

Proof. Suppose first that $\omega(G) / \zeta(G)$ is finite and that $x \tau(G) \in \omega(H)$. We shall prove that some power of $x$ is in $\omega(G)$. The result follows since then some power of $x$ is in $\zeta(G)$, by our hypothesis, and $\zeta(G) \tau(G) / \tau(G) \leq \zeta(H)$.

Since $G$ is polycyclic, there is an upper bound on the minimal number of generators of any subgroup of $G, m$ say. Now suppose that $S$ is a subnormal subgroup of $G$. Then $S_{0}=S \tau(G) / \tau(G)$ is subnormal in $H$ and $S_{0}^{x}=S_{0}$. Now $\left|S_{0}: S\right| \leq|\tau(G)|$. In any group with minimal number of generators at most $m$, there are a bounded number of subgroups of index at most $|\tau(G)|, M$ say. (See, for example, Problem 19, page 102 of Magnus, Karass and Solitar [9].) Then $x$ will act by conjugation as a permutation of the subgroups of index $\left|S_{0}: S\right|$ in $S_{0}$. In particular, $y=x^{M !}$ will act trivially on this set and so $S^{y}=S$. It follows that $x^{M !} \in \omega(G)$, as required.

Now suppose that $\omega(H) / \zeta(H)$ is finite. If $x \in \omega(G)$, then we have $(x \tau(G))^{m} \in \zeta(H)$, by our hypothesis. It will therefore be enough to show that if $y \tau(G) \in \zeta(H)$ then $y^{n} \in \zeta(G)$, for some $n$. The inner automorphism induced by $y$ acts on $G / \tau(G)$ trivially and so, by Lemma 2, $y$ induces an inner automorphism of finite order on $G$. The result follows.

The next two results enable us to show that under certain very restricted conditions we can show that some power of an automorphism is inner; our aim in the proof of Theorem 2 will be to reduce the general case to that of the lemma below. First we shall need a result about integral representations of a cyclic group of prime order $p$. Suppose that $X=\langle x\rangle$ is cyclic of order $p$. We denote by $\mathbb{Z}$ the ring of integers, by $\mathbb{Z}_{q}$ the field of $q$ elements, where $q$ is a prime, and by $\mathbb{Q}$ the field of rational numbers. We need some facts about $\mathbb{Z}_{p} X$ that are well known but not easily accessible. 
Observe that $\mathbb{Z}_{p} X$ is isomorphic to the quotient ring $\left.\mathbb{Z}_{p}[x] /\left(x^{p}-1\right) \mathbb{Z}_{p}[x]\right)$. Regarded as a $\mathbb{Z}_{p} X$-module, submodules of $\mathbb{Z}_{p}[x] /\left(\left(x^{p}-1\right) \mathbb{Z}_{p}[x]\right)$ correspond to ideals and these are just $(x-1)^{i} \mathbb{Z}_{p}[x] /\left(\left(x^{p}-1\right) \mathbb{Z}_{p}[x]\right)$, for $0 \leq i \leq p$, (noting that $\left.x^{p}-1=(x-1)^{p}\right)$. In particular, submodules and quotient modules are indecomposable and determined by their dimensions. (For another treatment of this, see Alperin [1], Corollary 1.3.3 and pages 24-25.)

Lemma 4. Let $R=\mathbb{Z} X$. Suppose that $W$ is an $R$-module that is free as abelian group and generated as module by one element. If $E=\operatorname{End}_{R}(W)$, then since $R$ is commutative we can consider $x$ as an element of $E$. Suppose that $1+\ldots+x^{p-1}=0$ in $E$. Then $W$ is an indecomposable $R$-module and $W$ has rank $p-1$ as free abelian group. Further, if $E_{t}$ is the endomorphism ring of the $R$-module $W / p^{t} W$, then $E_{t}$ is a local ring.

Proof. Since $W / p W$ is generated by a single element as $\mathbb{Z}_{p} X$-module, it is isomorphic to a quotient of $\mathbb{Z}_{p} X$ and hence is indecomposable. It follows that $W$ is an indecomposable $R$-module. But now, regarding $x$ as a linear transformation of $\mathbb{Q} \otimes W$ we have that its minimum polynominal must divide $1+\lambda+\ldots+\lambda^{p-1}$, (since it must also divide $\lambda^{p}-1$ ). But this is the $p^{\text {th }}$ cyclotomic polynominal and hence is irreducible over $\mathbb{Q}$. (See Lang [8, p. 316].) Thus $x$ has minimum polynomial $1+\lambda+\ldots+\lambda^{p-1}$ and so $\mathbb{Q} \otimes W$ has dimension $p-1$. It follows that $W$ has rank $p-1$ as free abelian group. Moreover $W / p^{t} W$ is indecomposable and hence, by Proposition 9.4 of Lang [8], $E_{t}$ is a local ring.

We now use Lemma 4 to show that under certain conditions some power of an automorphism will be inner. We denote the natural homomorphism from a group $G$ onto $\operatorname{Inn}(G)$ by $\theta_{G}$, and if the context is clear, by $\theta$.

Lemma 5. Let $G$ be a polycyclic group with $\tau(G)=1$, normal subgroups $M$ and $H$, with $M$ abelian, $M \leq H$ and $|G / H|$ finite. Let $1 \neq \alpha \in \operatorname{Aut}(G)$. Suppose that every subnormal subgroup $S$ satisfying $H \leq S \leq G$ is $\alpha$-invariant, and that $\alpha$ centralises $H$ and $G / M$. Then, for some integer $n$, we have $\alpha^{n}=\theta(m)$, for some $m \in M$.

Proof. We prove the result by induction on $|G / H|$. We show first that if $|G / H|=p$, for some prime $p$, there exists $v \in M$ such that $\alpha^{p^{2}}(g)=g^{v}$, for all $g \in G$.

If $x$ is an element of $G$ not in $H$, then we have $\alpha(x)=x w$, with $w \in M$. Note that $w \neq 1$ since then $\alpha$ would be the identity automorphism. Since $M$ is a free abelian group, we can regard it as a $\mathbb{Z}\langle x\rangle$-module. Let $W$ be the submodule generated by $w$. We note that $\alpha\left(x^{p}\right)=x^{p}=(x w)^{p}$ and so $x^{p}=x^{p} w w^{x} \ldots w^{x^{p-1}}$ or $w^{1+x+\ldots+x^{p-1}}=1$. Hence we get $w^{x^{p}}=w$ and so $\langle x\rangle$ acts on $W$ as a cyclic group of order $p$. It now follows from Lemma 4 that $W$ is indecomposable and of rank $p-1$.

We now show that $\alpha^{p}(x)$ is $x^{u}$, for some $u \in W$. To see this it is enough to show that $\alpha^{p}(x)$ and $x$ are conjugate in every finite quotient of $W\langle x\rangle$, by Theorem 3 of [12, p. 59], and to prove this it is enough to show they are conjugate in $W\langle x\rangle$ modulo $W^{n}$, for every integer $n$. If $n=p^{a} q$, with $q$, $p$ coprime, then it is an easy consequence of the Sylow theorems that if $\alpha^{p}(x)$ and $x$ are conjugate modulo $W^{p^{a}}$ then they are conjugate modulo $W^{p^{a} q}$, and so it is enough to show that $\alpha^{p}(x)$ and $x$ are conjugate modulo $W^{p^{t}}$, for all positive integers $t$.

By Lemma 4 , we have that $W / W^{p}$ is a $(p-1)$-dimensional $\mathbb{Z}_{p}\langle x\rangle$-module and so is uniqely determined. We consider the element $(1-x)^{p-1}$ as an element of 
$\operatorname{End}_{\mathbb{Z}\langle x\rangle}(W)$. We have $(1-x)^{p-1}=1-(p-1) x+\ldots+(-1)^{p-1} x^{p-1}$. We then have, putting $x^{p-1}=-1-x-\ldots-x^{p-2}$, that $(1-x)^{p-1}=a_{1} x+a_{2} x^{2}+\ldots+a_{p-2} x^{p-2}$, where $a_{i}=\left((-1)^{i}(p-1) \ldots(p-i) / i !\right)-1$. Thinking of $a_{i}$ as a polynominal in $p$ we see that the constant term is 0 . Thus $a_{i}=p b_{i}$, for each $i$, and $a_{1}=p$, giving

$$
(1-x)^{p-1}=p\left(x+b_{2} x^{2}+\ldots+b_{p-2} x^{p-2}\right)=p f(x) .
$$

Note also that $1-(p-1)+\ldots-(p-1)+1=0$ (put $x=1$ in the expansion of $\left.(1-x)^{p-1}\right)$ and so $1+(p-1)-\ldots+(p-1)(p-2) / 2-(p-1)=p$. Thus we have $b_{1}+\ldots+b_{p-2}=1$ and so $f(x)$ is not in the unique maximal ideal of $E_{t}$. Thus $f(x)$ has an inverse in $E_{t}$. We then have $u^{(1-x)^{p-1}}=w^{p}$, where $u=w^{f(x)^{-1}}$. Now consider $x^{v}$, where $v=u^{(1-x)^{p-2}}$. We then have $x^{v}=x[x, v]=x v^{(1-x)}$ and $v^{(1-x)}=u^{(1-x)^{(p-1)}}=w^{p}$. Thus we have $\alpha^{p}(x)=x w^{p}=x^{v}$, as required. Set $\beta=\alpha^{p}$. Note that if $[v, x]=1$ then $\beta$ is the identity automorphism on $G$, and so is inner. Thus we assume that $[v, x] \neq 1$.

We now have that $\beta$ acts as conjugation by $v$ on $x$ and centralises $H$; we do not know the action of $v$ on $H$ however. Consider now $y \in H$. We have $y^{x^{-1}} \in H$ and so $y^{x^{-1}}=\beta\left(y^{x^{-1}}\right)=y^{\beta\left(x^{-1}\right)}=y^{x^{-v}}$. Hence $y=y^{[v, x]}$. Thus $[v, x] \in \zeta(H)$ and $\beta(x)=x[v, x]^{-1}$. We can now, as in the previous paragraph, find $z$ in the normal closure of $[v, x]$ in $G$ such that $\beta^{p}(x)=x^{z}$ and moreover we now have $z \in \zeta(H)$. It follows that $\beta^{p}\left(x^{n} a\right)=\left(x^{n} a\right)^{z}$, for any integer $n$ and any $a \in H$, and so $\beta^{p}=\alpha^{p^{2}}$ acts as an inner automorphism on $G$, proving the result in this case.

Now suppose that the result is true for $\left(G^{*}, M^{*}, H^{*}, \alpha^{*}\right)$ satisfying the hypotheses of the lemma with $\left|G^{*} / H^{*}\right|<|G / H|$. We have established the result for $H$ maximal in $G$ and so we may suppose that there is a normal subgroup $K$ of $G$ satisfying $H<K<G$. It is easy to check that $(K, H, M, \alpha)$ (with $\alpha$ regarded as an automorphism of $K$ ) satisfies the hypotheses of the Lemma and hence, by our inductive hypothesis, for some integer $t$ we have $\alpha$, regarded as an automorphism of $K$, is such that $\alpha^{t}$ acts as the inner automorphism of $K$ induced by $z$, for some $z \in M$. Now consider $\beta=\alpha^{t} \theta(z)^{-1}$. Since $z \in M, \theta(z)$ centralises $G / M$ and so $\beta$ centralises $G / M$. Since $z \in S$, for any subnormal subgroup $S$ satisfying $K \leq S \leq G, S^{\beta}=S$. By its definition, $\beta$ centralises $K$. Thus we see that $(G, K, M, \beta)$ satisfies the hypotheses of the lemma. Then, by our inductive hypothesis, there is an integer $u$ and an element $y \in M$ such that $\beta^{u}=\theta(y)$. We then have $\left(\alpha^{t} \theta(z)^{-1}\right)^{u}=$ $\alpha^{t u} \theta(z)^{\alpha^{t(u-1)}} \ldots \theta(z)=\alpha^{t u} \theta\left(\alpha^{t(u-1)}(z) \ldots z\right)=\alpha^{t u} \theta(x)$, with $\quad x=\alpha^{t(u-1)}(z) \ldots z \in M$, since $M$ is $\alpha$-invariant. Thus finally we have $\alpha^{t u}=\theta\left(y x^{-1}\right)$ and the proof is complete.

2. Proof of Theorem 2. We consider first the case that $G$ is a polycyclic group with $\omega(G) / \zeta(G)$ finite; we shall show that then $\operatorname{Aut}_{s n}(G)$ is also finite and thus establish Theorem 1 as a special case of Theorem 2. Throughout the proof, we set $A=\operatorname{Aut}_{s n}(G)$.

Consider $H=G / \tau(G)$. By Lemma 3, $\omega(H) / \zeta(H)$ is finite and we can regard $A$ as acting on $H$. If $\operatorname{Aut}_{s n}(H)$ is finite, then $A / C_{A}(H)$ is also finite and so $A$ is finite, by Lemma 2. Thus it will be enough to consider the case in which $\tau(G)=1$. In this case it follows from Lemma 1 that $\omega(G)=\zeta(G)$.

If $A$ is infinite, then it is abelian, by Franciosi and de Giovanni [5, Theorem B] and finitely generated by Theorem 1 of $[\mathbf{1 2}$, p. 25]. Suppose that $\alpha$ is an element of $A$ of infinite order. We have, by Lemma 2.1 of Franciosi and de Giovanni [5], that $\alpha$ acts trivially on $G / \omega(G)$. Since $F(G)$, the Fitting subgroup of $G$, is a torsion-free 
nilpotent group on which $\alpha$ acts as a power automorphism, (since every subgroup of $F(G)$ is subnormal in $G$ ), we have that $\alpha^{2}$ acts trivially on $F(G)$ (and so in particular on $\zeta(G)$ ). We set $\beta=\alpha^{2}$. Now suppose that $x \in G$ is an element not fixed by $\beta$. Then $\beta(x)=x z$, for some $z \in \zeta(G)=\omega(G)$. If $x^{n} \in F(G)$, for some integer $n$, then we have $\beta\left(x^{n}\right)=x^{n}=(x z)^{n}=x^{n} z^{n}$ and so $z^{n}=1$, giving $z=1$, a contradiction. Hence $x$ is an element of infinite order and $\langle x\rangle \cap F(G)=1$. Since $G / F(G)$ is abelian-by-finite we can, by replacing $x$ by a power if necessary, assume that $L=F(G)\langle x\rangle$ is subnormal in $G$; $L$ is clearly not nilpotent. If $L / F(G)^{p}$ were nilpotent for all $p$ in an infinite set of primes $\pi$, then $L / F(G)^{p}$ would be nilpotent of class at most the Hirsch length of $F(G)$, for each $p \in \pi$, and then, since $\cap_{p \in \pi} F(G)^{p}=1$ by a theorem of G. Higman [6], we would have $L$ nilpotent, a contradiction. Thus the set of primes $p$ for which $L / F(G)^{p}$ is nonnilpotent is infinite. Choose $p>2$ to be a prime such that $L / F(G)^{p}$ is not nilpotent and $z \notin F(G)^{p}$. Since $x$ acts on $F(G) / F(G)^{p}$ by conjugation, we have some power of $x$ centralises $F(G) / F(G)^{p}$. Now choose $m$ to be the smallest positive power of $x$ for which $x^{m}$ centralises $F(G) / F(G)^{p}$; note that $m>1$. If $m=p^{t} k$ with $k$ coprime to $p$, then $F(G)\left\langle x^{k}\right\rangle / F(G)^{p}$ is the subdirect product of an infinite cyclic group and a finite $p$-group, and so is nilpotent. But now we have $F(G)^{p}\left\langle x^{k}\right\rangle$ subnormal in $F(G)\left\langle x^{k}\right\rangle$ and so in $G$. We can consider $\beta$ as an automorphism of $F(G)^{p}\left\langle x^{k}\right\rangle / F(G)^{p}$ and then we have $\gamma=\beta^{2}$ centralises $F(G)^{p}\left\langle x^{k}\right\rangle / F(G)^{p}$. It follows that $\gamma\left(x^{k}\right)=x^{k}$ modulo $F(G)^{p}$. Thus we have $x^{k}=\gamma\left(x^{k}\right)=\beta^{2}\left(x^{k}\right)=\left(x z^{2}\right)^{k}=x^{k} z^{2 k}$ modulo $F(G)^{p}$ and thus $z^{2 k} \in F(G)^{p}$. Since $2 k$ is coprime to $p$, we have $z \in F(G)^{p}$, a contradiction. This contradiction completes the proof for the case in which $\omega(G) / \zeta(G)$ is finite.

We now suppose that $\omega(G) / \zeta(G)$ is infinite; it follows immediately that $A$ and $\omega(G)$ are abelian (Franciosi and de Giovanni [5, Theorem B], and Robinson [10, 13.3.9]). By Lemma 3 we have that if $H=G / \tau(G)$ then $\omega(H) / \zeta(H)$ is also infinite. Suppose that $\operatorname{Aut}_{s n}(H) /\left(\operatorname{Aut}_{s n}(H) \cap \operatorname{Inn}(H)\right)$ is finite. Then for $\alpha \in A$ there is an integer $m$ such that $\alpha^{m}$ acts on $H$ as an inner automorphism, say conjugation by $g \tau(G)$. We put $\beta=\alpha^{m} \theta\left(g^{-1}\right)$. Then $\beta$ acts trivially on $H$ and it follows from Lemma 2 that $\beta^{n}=1$, for some integer $n$, and so $\alpha^{m n}$ is an inner automorphism. It follows that $A /(A \cap \operatorname{Inn}(G))$ is finite. Thus we may as well assume that $\tau(G)=1$.

We now have $C_{1}=C_{G}(\omega(G))$ of finite index in $G$ by Theorem 1 of Cossey [3]. We now define $C_{i}$ inductively by $C_{i}=C_{C_{i-1}}\left(\omega\left(C_{i-1}\right)\right)$. Note that, since $\tau(G)=1$, we have $\tau\left(C_{i}\right)=1$ also. Since $\omega(G) \leq F(G)$, we have $\omega(G) \leq \omega(F(G))=\zeta(F(G))$ and hence $F\left(C_{1}\right)=F(G)$ and $\omega(G) \leq \omega\left(C_{1}\right) \leq \zeta(F(G))$. It now follows that $\omega\left(C_{i}\right) \leq \omega\left(C_{i+1}\right) \leq \zeta(F(G))$ and thus, for some integer $m$, we must have $\omega\left(C_{m}\right)=\omega\left(C_{m+1}\right)$. We then have $\omega\left(C_{m+1}\right) \leq \omega\left(C_{m}\right) \leq \zeta\left(C_{m+1}\right) \leq \omega\left(C_{m+1}\right)$ and hence $\omega\left(C_{m+1}\right)=\zeta\left(C_{m+1}\right)$. It now follows from the first part of the proof that $A / C_{A}\left(C_{m+1}\right)$ is finite. Thus it will be enough to show that some power of any automorphism in $C_{A}\left(C_{m+1}\right)$ is inner.

Observe that $\omega(G)$ is an abelian normal subgroup of $G$ contained in $C_{m+1}$ and $G / C_{m+1}$ is finite. Also we have that if $\alpha \in C_{A}\left(C_{m+1}\right)$, then $\alpha$ centralises both $C_{m+1}$ and $G / \omega(G)$, and normalises every subnormal subgroup of $G$. It is then an immediate application of Lemma 5 to conclude that some power of $\alpha$ is an inner automorphism. This completes the proof of the theorem.

\section{REFERENCES}

1. J. L. Alperin, Local representation theory (Cambridge University Press, Cambridge, 1986). 
2. J. Cossey, Automorphisms fixing every subnormal subgroup of a finite group, Glasgow Math. J. 39 (1997), 111-114. $231-234$

3. J. Cossey, The Wielandt subgroup of a polycyclic group, Glasgow Math. J. 33 (1991),

4. M. Dalle Molle, Sugli automorphismi che fissano i sottogruppi subnormali di un gruppo risolubile, Boll. Un. Mat. Ital. A (7) 9 (1995), 483-491.

5. S. Franciosi and F. de Giovanni, On automorphisms fixing subnormal subgroups of soluble groups, Atti Accad. Naz. Lincei Rend. Fis. Mat. 8 (1988), 217-222.

6. G. Higman, Nilpotent groups, Proc. Amer. Math. Soc. 6 (1955), 284-285.

7. B. Huppert and N. Blackburn, Finite Groups II (Springer-Verlag, 1982).

8. S. Lang, Algebra (Second edition) (Addison Wesley, 1984).

9. W. Magnus, A. Karass and D. Solitar, Combinatorial Group Theory (Second revised edition), (Dover, New York, 1976).

10. D. J. S. Robinson, A course in the theory of groups (Springer-Verlag, 1980).

11. D. J. S. Robinson, Automorphisms fixing every subnormal subgroup of a finite group, Arch. Math. (Basel) 64 (1995), 1-4.

12. D. Segal, Polycyclic Groups (Cambridge University Press, Cambridge, 1983). 